\title{
The ClpP protease homologue is required for the transmission traits and cell division of the pathogen Legionella pneumophila
}

\author{
Xiang-hui Li, Yong-lun Zeng, Ye Gao, Xiao-cong Zheng, Qin-fen Zhang, Shi-ning Zhou, Yong-jun Lu*
}

\begin{abstract}
Background: Legionella pneumophila, the intracellular bacterial pathogen that causes Legionnaires' disease, exhibit characteristic transmission traits such as elevated stress tolerance, shortened length and virulence during the transition from the replication phase to the transmission phase. ClpP, the catalytic core of the Clp proteolytic complex, is widely involved in many cellular processes via the regulation of intracellular protein quality.

Results: In this study, we showed that ClpP was required for optimal growth of L. pneumophila at high temperatures and under several other stress conditions. We also observed that cells devoid of clpP exhibited cell elongation, incomplete cell division and compromised colony formation. Furthermore, we found that the clpPdeleted mutant was more resistant to sodium stress and failed to proliferate in the amoebae host Acanthamoeba castellanii.
\end{abstract}

Conclusions: The data present in this study illustrate that the ClpP protease homologue plays an important role in the expression of transmission traits and cell division of L. pneumophila, and further suggest a putative role of ClpP in virulence regulation.

\section{Background}

Legionella pneumophila, a Gram-negative, intracellular bacterial pathogen, is the opportunistic agent responsible for a severe form of pneumonia named Legionnaires' disease and the less severe flu-like Pontiac fever [1,2]. The remarkable capability of $L$. pneumophila to colonize a wide range of natural protozoa and mammalian host cells is mostly attributed to its unique Type IVB secretory system (T4BSS) whose components are encoded by the $d o t$ (defect in organelle trafficking) and $i c m$ (intracellular multiplication) genes [3-6]. L. pneumophila uses the Dot/Icm apparatus to inject effectors into the host cells to promote invasion and to modulate organelle trafficking, which in turn leads to formation of replication-permissive endosomes [7-9].

Similar to a variety of microbes, L. pneumophila undergoes a life cycle characterized by a biphasic conversion between a vegetative replicative form and a nonreplicating, infectious and stress resistant transmissive

\footnotetext{
*Correspondence: luyj@mail.sysu.edu.cn

Department of Biochemistry, School of Life Sciences, Sun Yat-sen University, Guangzhou 510275, China
}

form. On one hand, bacteria cultured in broth to either exponential or stationary phase display many similar attributes shared by the replicative and transmissive forms, respectively $[10,11]$. For example, upon the transition from exponential phase to stationary phase, $L$. pneumophila becomes more infectious and more resistant to various stresses [12]. Furthermore, L. pneumophila in stationary phase also displays shortened cell body, flagellin expression, pigment accumulation and reduced sodium sensitivity. These attributes, together with virulence markers such as cytotoxicity, intracellular growth and phagocytosis, are recognized as the transmission traits of $L$. pneumophila [11,13]. On the other hand, the in vitro-cultured stationary-phase L. pneumophila can achieve further differentiation to the cyst-like, hyper-infectious and resilient mature intracellular form (MIF) in aquatic environment or in specific mammalian cell lines. MIF is considered as an "in vivo stationaryphase form" while owning different outer membrane structure and protein composition compared with the stationary-phase form $[14,15]$. In addition, an in vivo transcriptome of L. pneumophila was performed and 
exhibited the genes strongly induced in intracellular replicative or transmissive phase, respectively, which also revealed several virulence or transmission related genes specially induced intracellularly, confirming the dissimilarity between the in vitro- and in vivo- transmissive/stationary phase [16].

A complicated gene network has been implicated in the regulation of transmission traits in L. pneumophila. For example, the sigma factor RpoS, the two-component system LetA/LetS, and the quorum sensing regulator LqsR have all been shown to facilitate the expression of transmission traits $[10,11,13,17,18]$. CsrA, a global repressor of transmission [19], also appears to be tightly regulated by several factors such as PmrA (positive regulator of several Dot/Icm-translocated effector proteins) and rsmYZ (two non-coding RNAs) [20,21]. In addition, CpxR has been found to activate transcription of several genes encoding components of the Dot/Icm complex as well as several Dot/Icm-translocated effectors [22,23]. The concerted action of these regulators not only contributes to the display of transmission traits, but also plays a vital role in the re-entry into the replicative phase [11,13,19,20,24].

Proteolysis of detrimental and misfolded proteins is critically important for protein quality control and cellular homeostasis [25-27]. Four classes of energy-dependent protease systems have been identified throughout prokaryotes: ClpAP/XP, ClpYQ (also named HslUV), FtsH and Lon. ClpP and ClpQ, the catalytic cores of the proteases, require Clp ATPase chaperones for the recognition and unfolding of substrates; on the other hand, in FtsH and Lon, a single polypeptide contains both ATPase and proteolytic activity $[26,28]$. The ClpP protease and Clp ATPase, which are widely distributed and highly conserved in various bacteria species as well as mitochondria and chloroplasts of eukaryotic cells $[27,29,30]$, have been demonstrated to function in the regulation of stress response, sporulation and cell division $[31,32]$. For example, ClpXP is responsible for the degradation of RpoS, the sigma regulator of stress response in E. coli [26]. In Salmonella enterica serovar typhimurium, loss of ClpXP has been shown to result in the over-expression of $f l i A$ and $f l i C$, which in turn induced a hyperflagellate phenotype [33]. In Bacillus subtilis, ComK/S, the two-component regulator of competence and sporulation, are tightly controlled by the successive binding and degradation mediated by MecA and ClpCP [26]. ClpP also seems to regulate virulence in many pathogens such as Listeria monocytogenes, Streptococcus pneumoniae and Staphylococcus aureus [31,34-36]. Finally, ClpP has been demonstrated to play a role in the biofilm formation [36-38].

As a ubiquitous bacterium in aquatic environment, $L$. pneumophila encounters numerous stresses such as elevated temperature, low $\mathrm{pH}$ and starvation during both planktonic existence and intracellular replication $[11,12]$. We hypothesized that a rapid response to a changing environment might require an uncharacterized proteolytic system in L. pneumophila. In the present study, we explored the role of L. pneumophila ClpP in growth, stress tolerance, cell morphology and virulence to amoebae host. We demonstrate that ClpP affects several L. pneumophila transmission traits and cell division, and ClpP might play an important role in virulence regulation.

\section{Results}

clpP homologue is required for optimal growth of $L$. pneumophila at high temperatures

In L. pneumophila, the lpg1861 sequence was predicted to encode a putative ClpP homologue. The product of lpg1861 consists of 215 amino acids and contains a highly conserved three-residue sequence Ser-His-Asp (Figure 1) that was previously reported as the proteolytic triad site of E. coli ClpP $[27,39,40]$. To investigate the physiological role of $c l p P$ homologue in L. pneumophila, we constructed a $c l p P$-deficient mutant by non-polar deletion of a 519 bp internal fragment encompassing the coding sequence for Ser-His-Asp. We first determined the impact of $c l p P$ on growth. As shown in Figure 2, the growth curves of WT, the Lp $\Delta$ clpP mutant, and the constitutive complemented strain $\mathrm{Lp} \Delta c l p P$-p $c l p P$, were similar at $25^{\circ} \mathrm{C}, 30^{\circ} \mathrm{C}$ and $37^{\circ} \mathrm{C}$ (Figure $2 \mathrm{~A}$ to $2 \mathrm{C}$ ), demonstrating that $c l p P$ is not required for optimal growth at lower temperatures. However, the Lp $\Delta$ clpP mutant strain exhibited impaired growth at $42^{\circ} \mathrm{C}$ relative to the other two strains (Figure 2D), indicating an important role of $c l p P$ homologue for optimal growth of L. pneumophila at high temperatures.

\section{clpP homologue is required for stress tolerance in stationary phase}

L. pneumophila can respond to various environmental stresses and cope with harsh conditions while entering eukaryotic hosts $[12,41]$. To assess whether clpP homologue may be involved in stress response, the above three strains were grown to logarithmic or stationary phase and exposed to various stress conditions. When the logarithmic-phase cells were exposed respectively to low $\mathrm{pH}$, hydrogen peroxide, potassium chloride, and heat shock, the survival rates of all three strains were similar and lower than those of the stationary-phase cells (data not shown). When treated with $\mathrm{pH} 4.0$ citric acid for 30 minutes, WT JR32 cells in stationary phase exhibited approximately $70 \%$ survival rate. However, only about $10 \%$ of Lp $\triangle$ clpP mutant cells survived (Figure $3 \mathrm{~A}$ ). Such a deficiency was rescued in the $\mathrm{Lp} \triangle \operatorname{clpP}$-pclpP strain (Figure $3 \mathrm{~A}$ ). This result indicated 


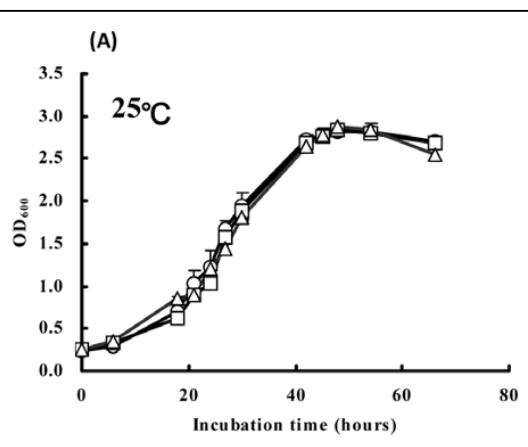

(C)

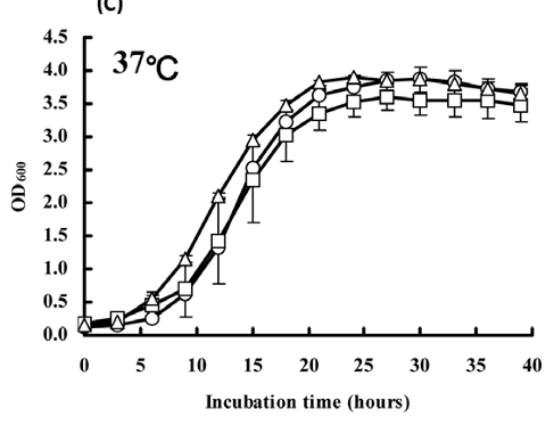

(B)

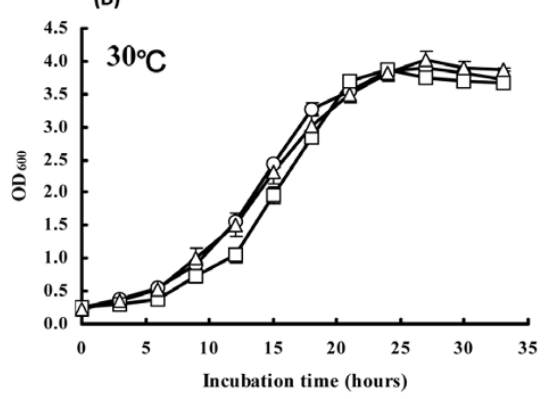

(D)

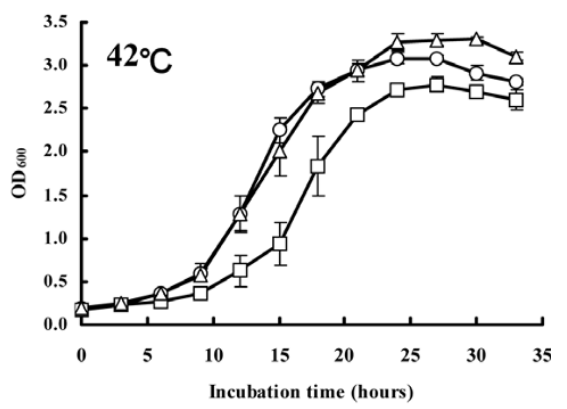

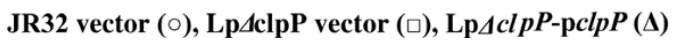

Figure 2 The growth curves of $L$. pneumophila wild-type JR32, the Lp $\Delta c / p P$ mutant, both harboring the vector pBC(gfp)Pmip, and the complemented strain $\mathbf{L} \mathbf{D} \boldsymbol{\Delta} \mathbf{c} \mathbf{p P} P$-pclpP. Overnight cultures of mid-exponential bacterial cells were diluted into fresh medium and then incubated at (A) $25^{\circ} \mathrm{C}$, (B) $30^{\circ} \mathrm{C}$, (C) $37^{\circ} \mathrm{C}$, and (D) $42^{\circ} \mathrm{C}$, respectively. Growth was monitored by $\mathrm{OD}_{600}$ at various time points. Points indicate mean values and error bars indicate standard deviations of three experiments.

that the deletion of $c l p P$ impairs the ability of L. pneumophila to respond to low-pH conditions. Similar results were also obtained in oxidative stress assay (Figure $3 \mathrm{~B}$ ). When the cells were treated with $1 \mathrm{mM}$ hydrogen peroxide for 30 minutes, the survival rate of the Lp $\triangle$ clpP mutant was $10 \pm 2.0 \%$, much lower than that of WT cells $(56 \pm 8.6 \%$; Figure $3 \mathrm{~B})$. In contrast, Lp $\Delta$ clpP-pclpP cells displayed a CFU closely resembling that of WT cells (Figure 3B). Likewise, when cells were incubated in $57^{\circ} \mathrm{C}$ water bath for 20 minutes or treated with $0.3 \mathrm{M}$ potassium chloride for 1 hour, the survival rate of $\mathrm{Lp} \Delta$ clpP mutant was lower than that of $\mathrm{WT}$ and the complementation strain (Figure $3 \mathrm{C}$ and $3 \mathrm{D}$ ), indicating that $c l p P$ is also required for responses to heat shock and osmotic stress. Collectively, these results indicate that ClpP homologue is involved in tolerance to multiple stresses in stationary-phase L. pneumophila.

\section{clpP homologue is required for normal cell division of $L$. pneumophila}

During stress tolerance assays, $\mathrm{Lp} \Delta c l p P$ generally exhibited 1.5 - to 3 -fold lower colony formation efficiency compared with WT JR32 on BCYE plates (data not shown). However, all three L. pneumophila strains appeared to have similar growth rates at $37^{\circ} \mathrm{C}, 30^{\circ} \mathrm{C}$ and $25^{\circ} \mathrm{C}$ (Figure $2 \mathrm{~A}$ to $2 \mathrm{C}$ ), thus excluding significant reduction in the number of living Lp $\Delta$ clpP cells. Previously, ablation of Clp protease activity has been shown to lead to abnormal cell wall formation or incomplete cell division in several Gram-positive bacteria [32]. To examine the morphology of Lp $\Delta$ clpP mutant cells under normal conditions, we performed cryo-transmission electron microscopy (cyro-TEM). Cells in stationary phase were frozen-hydrated by liquid nitrogen and directly observed at $-172^{\circ} \mathrm{C}$, and we found that $\mathrm{Lp} \Delta c l p P$ cell surface was surprisingly indistinguishable from that of the WT cells (Figure 4A and 4B), contrary to our results obtained by scanning electronic microscopy (SEM) (Figure 4D and 4E), indicating that ClpP deficiency did not affect cell wall architecture under normal growth conditions.

The combined results of SEM and cyro-TEM showed that unlike the "plump cocoid" shape of the WT or complemented strains, stationary-phase cells deficient in $c l p P$ were elongated and incapable to divide normally (Figure $4 \mathrm{~A}$ to $4 \mathrm{E}$ ). Furthermore, around $62 \%$ of $\mathrm{Lp} \Delta c l p P$ cells were twins, $23 \%$ were hyper-filamentous, and only $15 \%$ of cells were single (Figure $4 \mathrm{~F}$ ). In contrast, around 
(A)

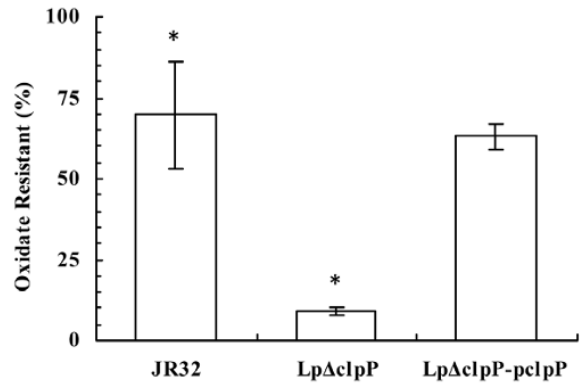

(C)

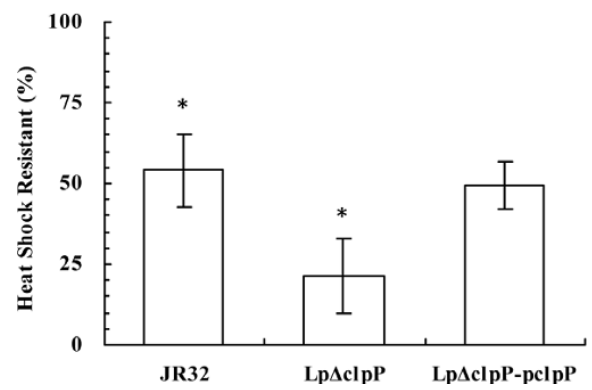

(B)

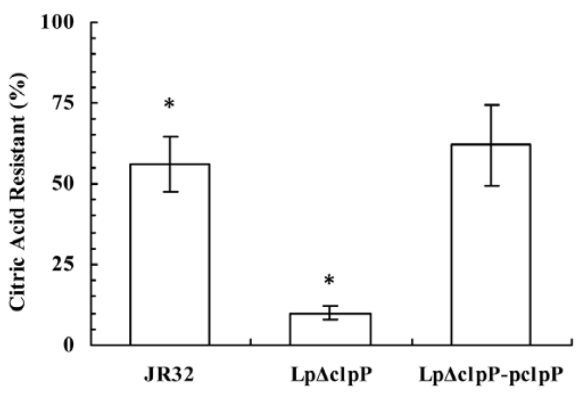

(D)

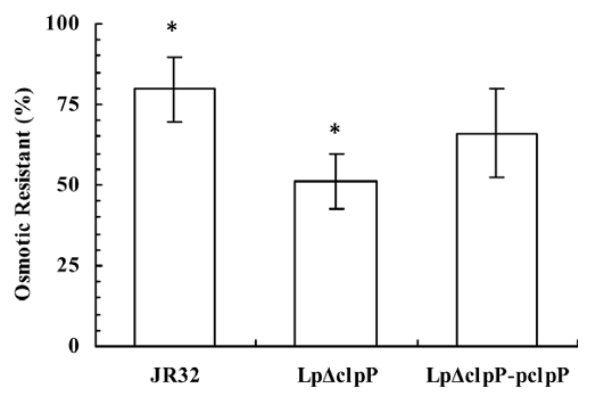

Figure 3 Impaired stress tolerance of the $L$. pneumophila $L \mathbf{L} \Delta \mathrm{clpP}$ mutant during stationary phase. Overnight cultures of different strains were inoculated into fresh medium and grew to stationary phase $\left(\mathrm{OD}_{600}\right.$ from 3.5 to 4.5), and the cells were then treated with $(\mathrm{A}) 1 \mathrm{mM} \mathrm{H}_{2} \mathrm{O}_{2}$ for 30 minutes. ${ }^{*} p<0.05$, (B) $\mathrm{pH} 4.0$ citric acid for 30 minutes. ${ }^{*} p<0.01$, (C) $57^{\circ} \mathrm{C}$ heat shock for 20 minutes. ${ }^{*} p<0.05$, or (D) $0.3 \mathrm{M} \mathrm{KCl}$ for 1 hour. ${ }^{*} p<0.05$. The experiments were carried out in triplicate.

$8 \%$ of WT JR32 cells were hyper-filamentous, and approximately $11 \%$ of cells were "twins" (Figure 4F). The abnormal cell morphology was also reversed by complementation (Figure $4 \mathrm{C}$ and $4 \mathrm{~F}$ ). These results together suggest that deletion of $c l p P$ lead to abnormal cell division and consequently aberrant cell morphology in L. pneumophila.

\section{The $L p \triangle c l p P$ mutant is sodium tolerant}

Stationary-phase L. pneumophila cells have been shown to exhibit sodium sensitivity $[42,43]$. It has been proposed that the assembly of virulence factor translocation apparatus, such as the Dot/Icm T4SS complex, allows high levels of sodium to diffuse into the cytoplasm, which is lethal to the cells [44]. To investigate whether ClpP homologue also affected sodium sensitivity of $L$. pneumophila, JR32, Lp $\Delta c l p P$ and Lp $\Delta c l p P$-pclpP strains were grown to exponential or stationary phase, diluted and plated in duplicate on BCYE or BCYE containing $100 \mathrm{mM}$ sodium chloride, respectively. Different dilutions of stationary-phase JR32 and Lp $\Delta$ clpP cells were also spotted on the plates. In the presence of sodium, exponential-phase cells exhibited indistinguishable sodium sensitivity, irrespective of the genotype (Figure 5A). However, the Lp $\triangle$ clpP mutant displayed an approximately 300-fold higher resistance than JR32 in stationary phase (Figure 5A). The loss of sodium sensitivity as a result of $c l p P$ deletion was again reversed in Lp $\Delta c l p P$-pclpP (Figure 5A). The relationship between sodium resistance and $c l p P$ deletion was further confirmed by the plate-spotting assay (Figure 5B). Notably, while more resitant to sodium in both assays, $\mathrm{Lp} \Delta c l p P$ required two more days to form colonies on $\mathrm{NaCl}$ plates compared to JR32 (Figure 5; data not shown). Taken together, these results demonstrate that the deletion of clpP enhances the sodium resistance of $L$. pneumophila in stationary phase with a slower growth rate, implying a possible role of $\mathrm{ClpP}$ in virulence.

\section{Loss of clpP impaires L. pneumophila growth and its cytotoxicity against $A$. castellanii}

To determine whether ClpP homologue may function in the virulence of $L$. pneumophila, we performed the amoebae plate test (APT) previously used to determine virulence [45]. The amoebae (A. castellanii) host cells were spread onto BCYE plates before stationary-phase L. pneumophila cells were spotted in 10-fold serial dilutions, and the plates were subsequently incubated at $37^{\circ}$ C for 5 days. As shown in Figure 6A, WT JR32 and the complemented strain Lp $\Delta$ clpP-pclpP exhibited robust growth even at $10^{-8}$ dilution when co-incubated with amoebae. However, Lp $\Delta c l p P$ showed a growth defect 

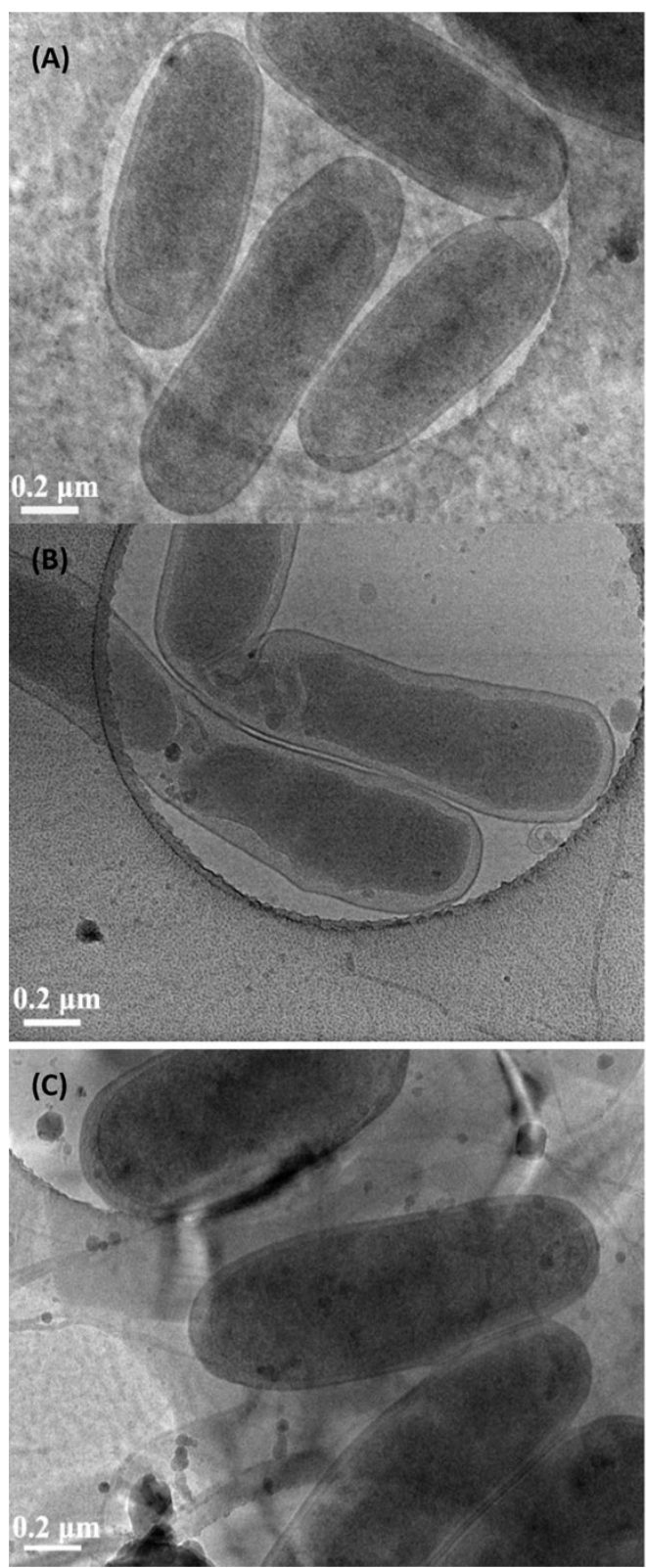
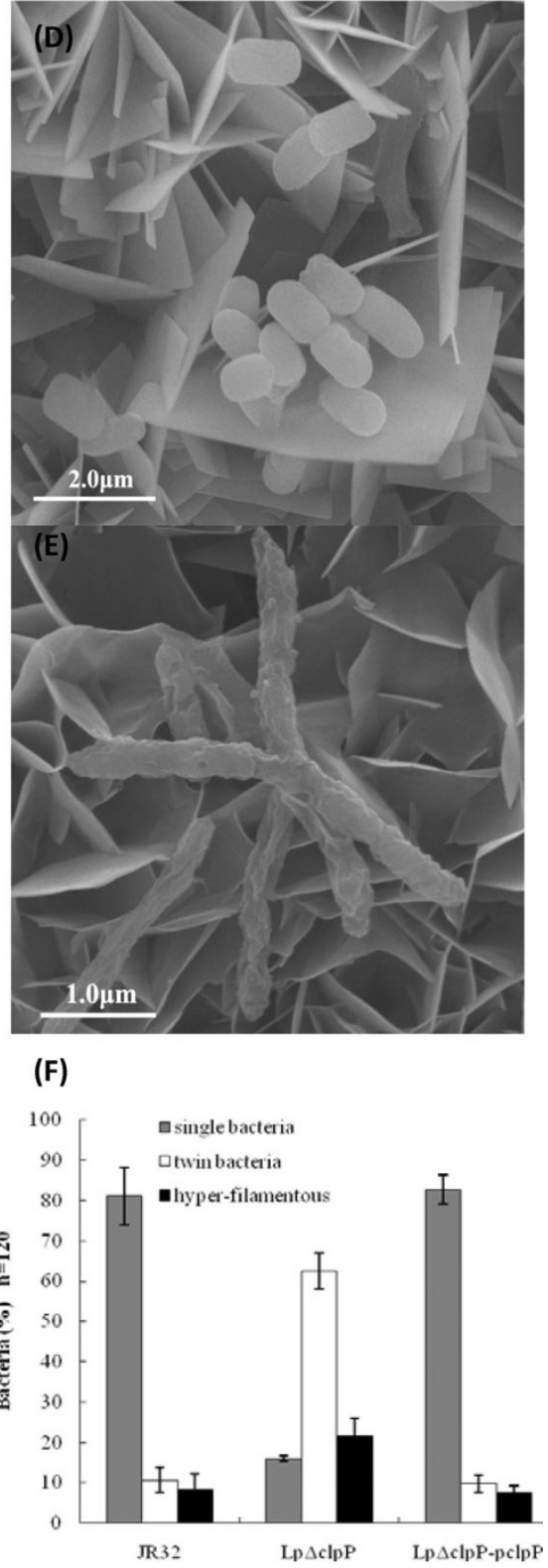

Figure 4 Electron microscopy of stationary-phase L. pneumophila cells revealed cell elongation and abnormal division in the $L p \Delta c l p P$

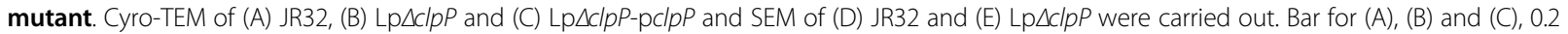
$\mu \mathrm{m}$; Bar for (D), $2.0 \mu \mathrm{m}$; Bar for (E), $1.0 \mu \mathrm{m}$. (F) The percentages of normal and abnormal cells under cyro-TEM in the three L. pneumophila strains. Shown are the averages and standard deviations of three independent counts and the number of cells for each count is about 120 ( $n=120$ ).

resembling the phenotype observed in the negative control $\Delta d o t A$ strain which was rendered completely avirulent by an in-frame deletion in the $\operatorname{dot} A$ gene [46]. As an additional control, cells were spotted onto the plates in the absence of amoebae, and no difference in growth was observed among the four strains (data not shown).

Cytotoxicity is an important virulent trait of L. pneumophila and correlates strongly with the function of the Dot/Icm T4SS [13,44,45,47]. We next tested whether
clpP homologue may affect the cytotoxicity of L. pneumophila against $A$. castellanii. L. pneumophila strains were used to infect $A$. castellanii with an MOI of 100. $24 \mathrm{~h}$ post infection, cytotoxicity was assayed by PI staining and quantified by flow cytometry analysis $[13,45]$. As shown in Figure 6B, JR32 exhibited robust cytotoxicity ( $70 \% A$. castellanii lethality), whereas $\mathrm{Lp} \Delta$ clpP resulted in only $17 \%$ cell death, barely higher than that of the avirulent mutant $\Delta \operatorname{dot} A$ ( $9 \%$ cell death). As expected, 
(A)

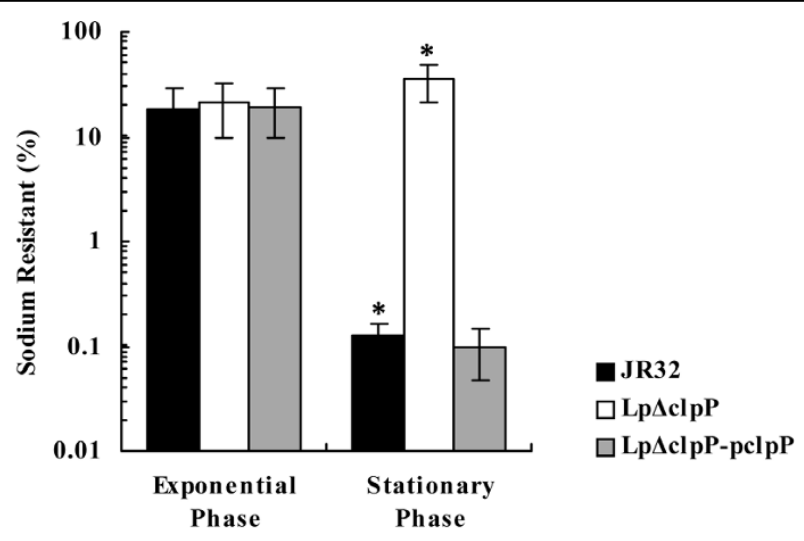

(B)

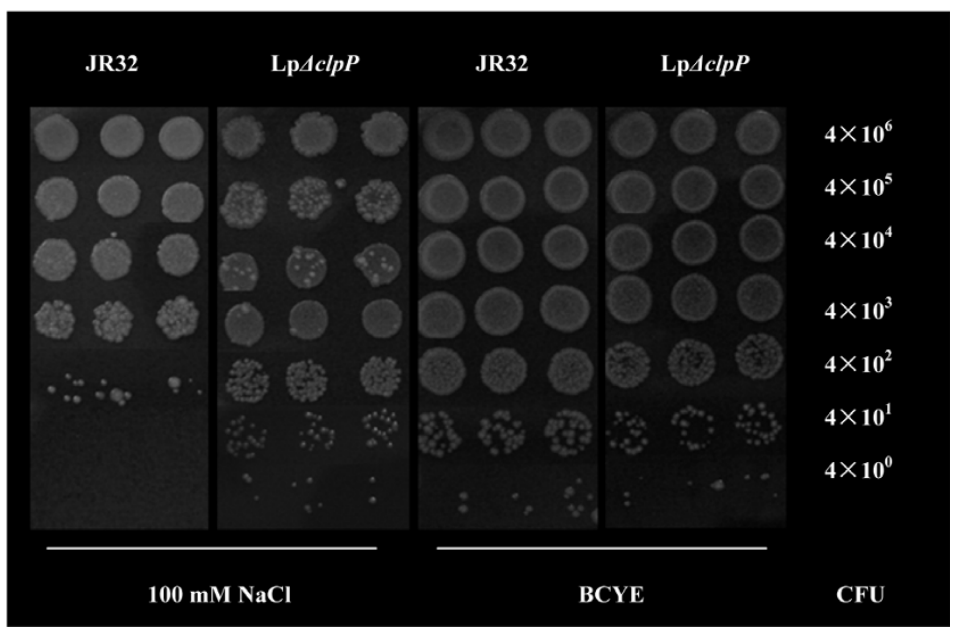

Figure 5 Sodium tolerance of $L$. pneumophila $L \mathbf{L} \Delta c \mid p P$ mutant was enhanced. (A). Overnight bacterial cultures in mid-exponential phase were inoculated into fresh medium and grew to exponential phase $\left(\mathrm{OD}_{600}\right.$ from 1.0 to 1.5$)$ or stationary phase $\left(\mathrm{OD}_{600}\right.$ from 3.5 to 4.5$)$, then the CFU was determined by plating duplicate samples of JR32 (black bars), Lp $\triangle$ clpP mutant (white bars), and complemented strain (gray bars) on BCYE and BCYE containing $100 \mathrm{mM} \mathrm{NaCl}$. The experiment was carried out in triplicate. ${ }^{*} p<0.01$. (B). For direct visualization, different dilutions of stationary-phase JR32 and Lp $\triangle$ IPP cells were also spotted onto plates in triplicate.

cytotoxicity was restored in the complemented strain Lp $\Delta c l p P$-p $c l p P$ (67\% PI positive). These results were also confirmed by fluorescence microscopy (Figure 6C). Thus, it appeared that loss of $c l p P$ seriously impaires cytotoxicity against the amoebae host.

\section{Loss of $c l p P$ abolishes intracellular multiplication of $L$. pneumophila in $A$. castellanii}

The above APT and cytotoxicity assays demonstrated an important role of $\operatorname{clpP}$ in virulence. Next, we examined whether $\operatorname{clp} P$ homologue also affected the intracellular replication of L. pneumophila in A. castellanii. Amoebae cells were infected with stationary-phase $L$. pneumophila at an MOI of 10. Under such conditions, the infection persisted for 3 days and multiplication was evaluated by plating the amoebae lysate onto CYE plates to quantify replication. As shown in Figure 7, JR32 and the complemented strain exhibited essentially identical replicative capability within $A$. castellanii cells. In contrast, both Lp $\Delta c l p P$ and $\Delta d o t A$ mutants showed significantly impaired multiplication. As a control, the $\mathrm{Lp} \triangle \operatorname{clpP}$ strain displayed normal growth at $30^{\circ} \mathrm{C}$ or $37^{\circ}$ $\mathrm{C}$ in broth (Figures $2 \mathrm{~b}$ and $2 \mathrm{c}$ ).

Taken together, the adverse effects of $c l p P$ deletion on sodium tolerance, growth on APT, cytotoxicity and intracellular multiplication suggest that $\mathrm{ClpP}$ homologue 
(A)

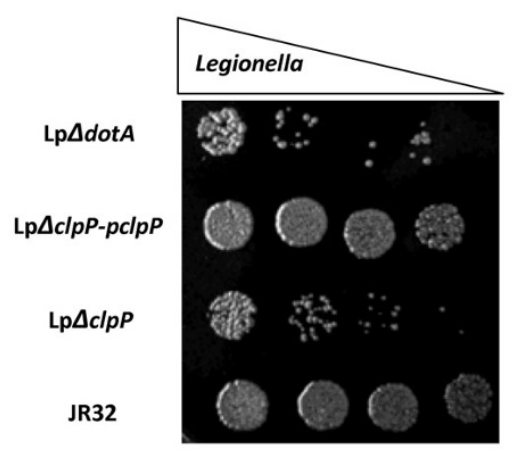

(B)

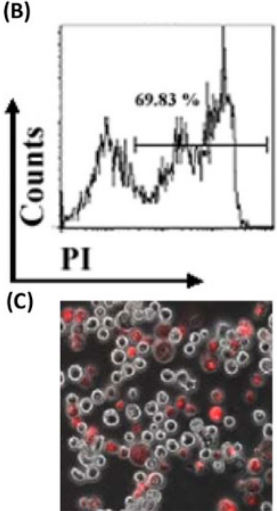

JR32
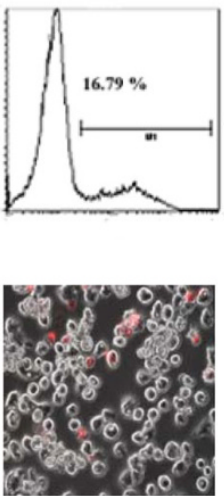

Lp $\Delta c / p P$
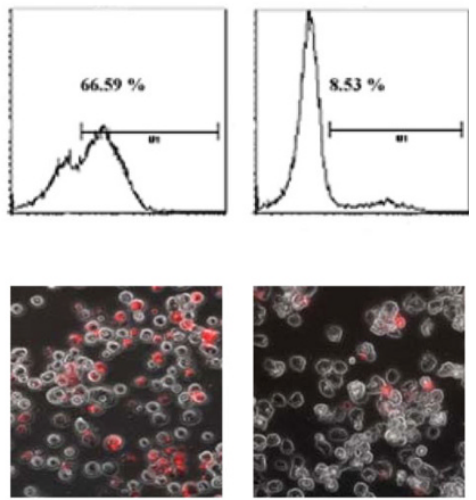

Lp$\Delta c / p P-p c l p P$

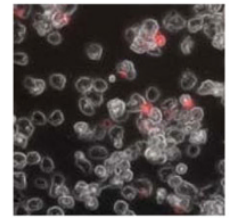

Lp$\Delta$ dotA

Figure 6 The L. pneumophila clpP mutant was impaired in both cytotoxicity against amoebae $A$. castellanii and growth on amoebae plates. (A) Growth of L. pneumophila Lp $\triangle \mathrm{c} / \mathrm{P} P$ mutant in the amoebae plate test was impaired. L. pneumophila wild-type strain JR32, Lp $\triangle c / p P$ mutant, $c / p P$ complemented strain or $\operatorname{dot} A$ mutant were spotted respectively in tenfold serial dilutions onto BCYE agar plates containing $A$. castellanii. The plates were incubated at $37^{\circ} \mathrm{C}$ for 5 days. (B) Cytotoxicity of $\mathrm{L}$. pneumophila against amoebae A. castellanii was quantified by flow cytometry and $(C)$ detected by PI staining $24 \mathrm{~h}$ post infection. The infection was performed using the wild-type strain JR32, Lp $\Delta c / p P$ mutant, clpP complemented strain or dotA mutant at an $\mathrm{MOI}$ of 100 . For fluorescence microscopy, amoebae cells in each well of 24-well plate were stained. The data shown are representative of at least two independent experiments.

might play an important role in virulence regulation of L. pneumophila.

\section{Discussion}

In the current study, Lp $\Delta c l p P$ was shown to exhibit reduced growth rate at high temperatures (Figure 2D) and impaired resistance to heat shock (Figure 3C) compared to the wild type. The $\mathrm{Lp} \Delta$ clpP mutant also displayed impaired resistance to oxidative and low- $\mathrm{pH}$ conditions in stationary phase. As oxidative and acid stress are generally considered as harsh and detrimental to DNA [48,49], ClpP homologue may play an important role in L. pneumophila DNA repair, consistent with its demonstrated function in E. coli [50], S. aureus [51] and Lactococcus lactis [52]. However, while several previous studies have demonstrated growth defect as a result of ClpP deficiency over a broad temperature range [34,35,51], deletion of $c l p P$ appeared to compromise the growth of L. pneumophila only at higher temperatures (Figure 2A to $2 \mathrm{C}$ ), suggestive of a more restricted role independent of cold response.

Attenuation of ClpP or Clp ATPase activities has been shown to lead to abnormal bacterial morphology such as filamentation, aberrant cell wall structure and irregular cell division [29,32,53-55]. Likewise, results from SEM and cyro-TEM revealed that the Lp $\Delta c l p P$ mutant cells were elongated and defective in cell division (Figure 4). Furthermore, SEM results also implicated a role of $c l p P$ in stress tolerance in L. pneumophila. In contrast to the defective cell surface observed in SEM (Figure 4D and $4 \mathrm{E}$ ), largely normal cell surface were found by cyroTEM in Lp $\triangle$ clpP mutant cells grown under normal 


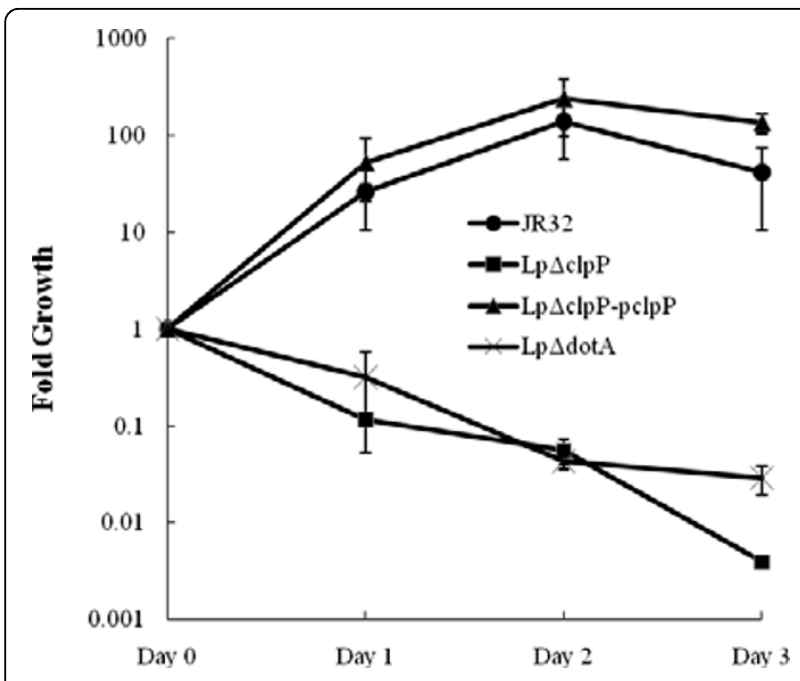

Figure 7 Intracellular growth of L. pneumophila Lp $\Delta c \mid p P$ mutant in $A$. castellanii was abolished. A. castellanii cells were seeded onto 24-well plates and infected with L.pneumophila at an $\mathrm{MOI}$ of 10. At each time point indicated, amoebae cells were lysed and the CFU was determined by plating dilutions onto BCYE plates. The intracellular growth kinetics of JR32, Lp $\triangle$ clpP mutant, clpP complemented strain, and $d o t A$ mutant are shown. The infection assay was carried out in triplicate.

conditions (Figure 4A to $4 \mathrm{C}$ ), suggesting that the chemical treatment during SEM sample preparation, not $\operatorname{clp} P$ deletion, may have resulted in the abnormal cell surface.

How ClpP affects cell division is not fully understood. In $C$. crescentus, degradation of the cell cycle repressor CtrA by the ClpXP complex has been shown to contribute to $\mathrm{G}_{1}-\mathrm{S}$ transition, and deletion of $c l p P$ blocked cell division [54]. In B. subtilis, cells overproducing MurAA, an enzyme in peptidoglycan biosynthesis and a substrate of the Clp protease, displayed a filamentous, undivided morphology reminiscent of the $\operatorname{clpP}$ mutant cells, suggesting that degradation of MurAA by ClpP might contribute to normal cell segregation [56]. Furthermore, through a ClpP-independent pathway, the B. subtilis ClpX appeared to modulate the assembly of the tubulin-like protein FtsZ [57], which is known to be a key process in the replication and division of Gramnegative bacteria [58]. Identification of the substrate(s) for ClpP may shed light on the regulatory mechanism of cell division in L. pneumophila.

ClpP proteolytic complexes play pivotal roles in protein degradation or modification [26,31,32]. During the transition of $B$. subtilis cells to stationary phase, ClpP degrades massive amounts of proteins previously produced in exponential growth phase [32]. Notably, $L$. pneumophila also undergoes a biphasic life cycle with mutually exclusive gene expression for replication or transmission $[10,11]$. While transiting from replication (exponential phase in vitro) to transmission (stationary phase in vitro), L. pneumophila activates an intricate network of regulators such as LetA/S, RpoS, PmrA, CpxR, rsmYZ, CsrA and LqsR [11,13,20,21,59]. As shown in our results, unlike the stationary-phase wild type which exhibits transmission traits, $\mathrm{Lp} \Delta c l p P$ mutant cells in stationary phase exhibit replicative forms such as reduced stress tolerance (Figure 2 and 3), cell elongation (Figure 4), enhanced sodium resistance (Figure 5), impaired cytotoxicity and growth on amoebae plates (Figure 6) and severely compromised intracellular multiplication in amoebae host (Figure 7). Thus, ClpP may play an important role in the transition from replication to transmission in L. pneumophila. On the other hand, several transmission traits are not affected by $c l p P$-deletion such as pigment accumulation and transcription from the flaA (legionella flagellin coding) gene (our unpublished data), suggesting that the impact of ClpP on the transition to transmissive form in L. pneumophila is somewhat limited. Considering that ClpP always executes the post-transcriptional feedback regulation, and moreover, degrades the same substrates by cooperating with other proteases [26,31], one explanation to such a limitation is that the degradation of ClpP substrates could be compensated by other proteases in clpP-deletion mutant, thus ClpP cannot govern the transition just as the global regulators such as RpoS, CsrA or LetA/S in L. pneumophila.

ClpP plays prominent roles in virulence of various Gram-positive pathogens such as S. aureus, S. pneumoniae and L. monocytogenes [34-36,60]. Furthermore, $\mathrm{ClpP}$ was reported to control the levels of key virulence factors of type III secretory systems (T3SS) in certain pathogens such as $S$. typhimurium and Yersinia pestis $[61,62]$. Recently, it was reported that loss of ClpP attenuated the virulence of Helicobacter pylori, a pathogen owning type IV secretory system (T4SS) [63]. It is interesting that $c l p P$-deletion severely compromised the $L$. pneumophila infection against amoebae host (Figure 6 and 7). In our results, the sodium resistance exhibited by $\mathrm{Lp} \Delta c l p P$ mutant (Figure 5 ), which is a phenotype shared by the mutants without functional Dot/Icm T4SS $[48,64]$, together with the comparable decline in intracellular multiplication observed in $\operatorname{Lp} \Delta \operatorname{clpP}$ and $\Delta \operatorname{dot} A$ mutants (Figure 7), suggest a role of ClpP in T4SSdependent virulence through degrading a repressor or activating an up-regulator of the substrate(s) of ClpP. One possibility is that the $\mathrm{ClpP}$ protease has a major impact on the expression or function of Dot/Icm T4SS in L. pneumophila. Another possibility is that ClpP might be required for the expression of some T4SS substrates. In this case, loss of ClpP would also severely attenuate the intracellular growth even if the T4SS is intact, just as the case of L. pneumophila Sigma S factor (RpoS) [59]. Thus, identification of the substrate(s) of 
$\mathrm{ClpP}$, which is currently underway in our laboratory, would help to discern the underlying relationship between ClpP and T4SS-dependent virulence in $L$. pneumophila.

\section{Conclusions}

In summary, our study shows that the L. pneumophila $\mathrm{ClpP}$ homologue is required for cell division and several transmission traits including stress tolerance, cell shortening, sodium sensitivity, cytotoxicity, growth on amoebae plates and intracellular multiplication. The study further suggests that the ClpP homologue might be important for virulence regulation of L. pneumophila.

\section{Methods}

\section{Cells and reagents}

The bacterial strains, plasmids and primers used in this work are listed in Table 1. Legionella pneumophila strains were cultured on buffered charcoal yeast extract (BCYE) plates, or in $\mathrm{N}$-(2-acetamido)-2-aminoethanesulfonic acid (ACES)-buffered yeast extract (AYE) medium, supplemented with $5 \mu \mathrm{g}$ chloramphenicol ml${ }^{-1}$ if necessary [65]. Escherichia coli strains were cultured in LuriaBertani (LB) agar plates or broth, supplemented with 30 $\mu \mathrm{g}$ chloramphenicol $\mathrm{ml}^{-1}$ or $100 \mu \mathrm{g}$ ampicillin $\mathrm{ml}^{-1}$.
Acanthamoeba castellanii (ATCC 30234) was grown in proteose yeast extract glucose medium (PYG) at $30^{\circ} \mathrm{C}$ [66]. Bacto yeast exact and proteose peptone were obtained from Becton Dickinson Biosciences. All other reagents were from Sigma, unless specified otherwise.

\section{DNA manipulation and chromosomal in-frame deletion}

DNA manipulations were performed according to standard protocols [67]. All restriction enzymes were purchased from New England Biolabs. Pfu or Taq DNA polymerases were from TaKaRa. Purification of plasmids and genomic DNA was performed according to the manufacturer's instructions (Qiagen).

The in-frame deletion of $\operatorname{clp} P$ was performed by a non-polar strategy as described [68]. Briefly, upstream and downstream flanking sequences of $c l p P$ were amplified by $\mathrm{PCR}$ using the $\mathrm{P}_{\mathrm{XC}-\mathrm{F} 1} / \mathrm{P}_{\mathrm{XC}-\mathrm{R} 1}$ and $\mathrm{P}_{\mathrm{XC}-\mathrm{F} 2} / \mathrm{P}_{\mathrm{XC}-\mathrm{R} 2}$ primer pairs, respectively. The PCR products were mixed and then used as templates for the subsequent fusion PCR using the $\mathrm{P}_{\mathrm{XC}-\mathrm{F} 1} / \mathrm{P}_{\mathrm{XC}-\mathrm{R} 2}$ primers. Fusion PCR products were digested with KpnI and $S a c I$ and sub-cloned into the pRE112 suicide vector [69], yielding plasmid pRE $\Delta c l p P$. Allelic exchange was performed as follows. Briefly, pRE $\triangle \operatorname{clp} P$ was introduced into the wildtype (WT) JR32 strain by electroporation and

Table 1 Bacterial strains, plasmids and oligonucleotides used in this study.

\begin{tabular}{|c|c|c|}
\hline $\begin{array}{l}\text { Strain, plasmid or } \\
\text { primer }\end{array}$ & Phenotype, genotype or sequence & $\begin{array}{l}\text { Reference or } \\
\text { source }\end{array}$ \\
\hline \multicolumn{3}{|l|}{ E.coli strains } \\
\hline $\mathrm{DH} 5 \alpha$ & 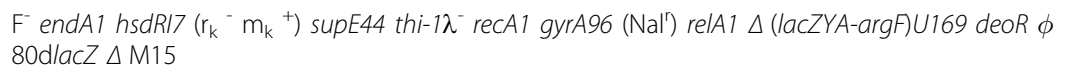 & Lab collection \\
\hline DH5 $\alpha \lambda$ pir & $\mathrm{DH} 5 \alpha$ transduced with $\lambda$ pir & {$[69]$} \\
\hline \multicolumn{3}{|c|}{ L. pneumophila strains } \\
\hline $\mathrm{JR} 32$ & Virulent L. pneumophila serogroup 1, strain Philadelphia, salt-sensitive isolate of AM511 & {$[43]$} \\
\hline $\mathrm{Lp} \Delta c / p P$ & JR32 with clpP deletion & This study \\
\hline $\mathrm{Lp} \Delta c \mid p P-p c l p P$ & $\mathrm{Lp} \Delta c / p P$ containing $p c l p P$ & This study \\
\hline JR32-pBC & JR32 containing pBC(gfp)Pmip & This study \\
\hline $\mathrm{Lp} \triangle c / p P-\mathrm{pBC}$ & Lp $\triangle c / p P$ containing pBC(gfp)Pmip & This study \\
\hline Lp $\triangle$ dotA & JR32 with $\operatorname{dot} A$ deletion & Lab collection \\
\hline \multicolumn{3}{|l|}{ Plasmids } \\
\hline pRE112 & Mobilizable suicide vector for construction of gene knockouts in $\mathrm{G}^{-}$bacteria, oriT oriV sacB Cm & {$[69]$} \\
\hline pMD18-T & cloning vector, Ap & TaKaRa \\
\hline pBC(gfp)Pmip & ColE1 ori Cm Pmip gfpmut2 & {$[70]$} \\
\hline $\mathrm{pRE} \triangle c / p P$ & pRE112::clpP for $c l p P$ deletion & This study \\
\hline $\mathrm{pclp} P$ & pBC(gfp)Pmip containing clpP under the control of mip promoter & This study \\
\hline \multicolumn{3}{|l|}{ Primers } \\
\hline$P_{X C-F 1}$ & AGAGAGCTCCTGCCAGTAGGTCCTATAAG & This study \\
\hline$P_{X C-R 1}$ & TATGACATACAAGTTGCTGGACATTCTAC & This study \\
\hline$P_{X C-F 2}$ & CAACTTGTATGTCATAGGAACGCTCACC & This study \\
\hline$P_{X C-R 2}$ & GATGGTACCTGGGAAAATTGACAAACCGT & This study \\
\hline$P_{X H-c l p P F}$ & TGGTGGAAGCTITAGGAGTATCTAGCAAAGTTATAAGTC & This study \\
\hline$P_{X H-c l p P R}$ & TGGTGGTCTAGATGAGAAAAAAGGAGAGTAAGC & This study \\
\hline
\end{tabular}

${ }^{*}$ Abbreviations: $\mathrm{Ap}$, ampicillin resistant; $\mathrm{Cm}$, chloramphenicol resistant; sacB, sucrose sensitive. 
chloramphenicol $^{\mathrm{R}+}$ colonies were selected on BCYE-Cm plates. Transformants were inoculated into AYE and then incubated on BCYE containing 5\% sucrose for 3 days at $37^{\circ} \mathrm{C}$ to select for strains devoid of the vector backbone. Positive colonies were confirmed by PCR and sequencing.

\section{Complementation assay}

A ColE1-type plasmid pBC(gfp)Pmip, carrying an enhanced GFP gene (gfpmut2) whose transcription was controlled by Pmip, the promoter of the Legionella-specific mip (macrophage infectivity potentiator) gene, was used for the $\operatorname{clp} P$ compensation experiment [70,71]. As a control, the transcriptional activity of the mip promoter was not discernibly affected by the loss of $c l p P$ in JR32 (data not shown). pBC(gfp)Pmip was digested with $X b a \mathrm{I}$ and HindIII to remove the $g f p$. Sequences of $c l p P$ were amplified by $\mathrm{PCR}$ using the $\mathrm{P}_{\mathrm{XH}-\mathrm{clp} P F}$ and $\mathrm{P}_{\mathrm{XH}-\mathrm{clpPR}}$ primers, and the products were digested with $\mathrm{XbaI}$ and HindIII. The digestion products were ligated with the vector. The constructed plasmid pclpP was then electroporated into $\mathrm{Lp} \triangle \mathrm{clp} P$, providing exogenous expression to compensate for the loss of $c l p P$.

\section{Growth experiments}

The growth experiments were conducted using three $L$. pneumophila strains, including JR32 and the $c l p P$ deficient $\mathrm{Lp} \triangle c l p P$ derivative, both harboring the $\mathrm{pBC}(\mathrm{gfp})$ Pmip vector, as well as the complemented strain $\mathrm{Lp} \Delta c l p P$-p $c l p P$. These strains were first grown in $5 \mathrm{ml}$ AYE for about $20 \mathrm{~h}$. The cultures were expanded into $30 \mathrm{ml}$ AYE in flasks, incubated to mid-exponential phase [optical density at $600 \mathrm{~nm}\left(\mathrm{OD}_{600}\right)$ 1.5-2.5], then diluted into new flasks to similar optical densities at approximate $\mathrm{OD}_{600} 0.2$. These new cultures were then incubated at $25^{\circ} \mathrm{C}, 30^{\circ} \mathrm{C}, 37^{\circ} \mathrm{C}$, and $42^{\circ} \mathrm{C}$, respectively. $\mathrm{OD}_{600}$ was determined by Beckman Du-530 at various time points.

\section{Stress resistance assays}

Resistance to stresses was measured as previously described $[12,65]$, with minor modifications. Cells from $1 \mathrm{ml}$ broth cultures were centrifuged at 5,000 g for 5 min, and resuspended in AYE supplemented with $1 \mathrm{mM}$ hydrogen peroxide, $0.1 \mathrm{M}$ citric acid at $\mathrm{pH} 4.0$, or $0.3 \mathrm{M}$ potassium chloride, respectively. $30 \mathrm{~min}$ later $(1 \mathrm{~h}$ for osmotolerance assay), cells were washed by centrifugation and resuspended in AYE. Cultures were subsequently serially diluted in water, plated on BCYE for colony forming unit (CFU) counting.

In heat resistance assays, cells from $1 \mathrm{ml}$ broth cultures were centrifuged at $5,000 \mathrm{~g}$ for $5 \mathrm{~min}$ and then resuspended in AYE. Samples for heat-shock were placed in a $57^{\circ} \mathrm{C}$ water bath for $20 \mathrm{~min}$, with the control in a $37^{\circ} \mathrm{C}$ water bath. Cells were washed and serially diluted in AYE, and spread on BCYE for CFU counting.

Stress resistance was calculated as [(stressed sample $\left.\mathrm{CFU} \mathrm{ml}^{-1}\right) /\left(\right.$ control sample CFU ml $\left.\left.\mathrm{m}^{-1}\right)\right] \times 100$.

\section{Sodium sensitivity assay}

Sodium sensitivity assay was performed as previously described [65]. Briefly, cells from $1 \mathrm{ml}$ broth cultures were centrifuged at $5,000 \mathrm{~g}$ for $5 \mathrm{~min}$ and then resuspended in AYE. Subsequently, the cell suspensions were serially diluted in water, and spotted on BCYE and BCYE containing $100 \mathrm{mM} \mathrm{NaCl}$ or spread on plates for CFU counts. Sodium sensitivity was calculated as [(BCYE-100 $\left.\left.\mathrm{mM} \mathrm{NaCl} \mathrm{CFU} \mathrm{ml}{ }^{-1}\right) /\left(B C Y E C F U ~ l^{-1}\right)\right] \times 100$.

\section{Electron microscopy}

For scanning electron microscopy (SEM), L. pneumophila cells in exponential or stationary phase were collected by centrifugation at $5,000 \mathrm{~g}$ for 2 minutes, and then washed 3 times with $1 \times \mathrm{PBS}$. After being fixed by $2 \%$ glutaraldehyde ( $\mathrm{pH} 7.4$ ) and $1 \%$ osmium tetroxide followed by dehydration in a graded ethanol series and isoamyl acetate embedding, the cells were dried by using a critical point drying method, and mounted on aluminum stubs and shadowed with gold. For visualization, a scanning electron microscope (Hitachi/Oxford S-520/ INCA 300) was used at $10 \mathrm{kV}$.

For Cryo-transmisson electron microscopy, L. pneumophila cells were collected and washed using the same method as above. The cells were then resuspended in $1 \times \mathrm{PBS}$ and $4 \mu \mathrm{l}$ sample aliquots were directly applied to a holey carbon film grid (R3.5/1 Quantifoil Micro Tools $\mathrm{GmbH}$, Jena, Germany), followed by blotting with filter paper (Whatman \#1) for about 3 seconds. The grid was then immediately flash frozen by plunging into pre-cooled liquid ethane. The cryo-grid was held in a Gatan 626 Cryo-Holder (Gatan, USA) and transferred into TEM (JEOL JEM-2010 with $200 \mathrm{kv} \mathrm{LaB}$ filament) at $-172^{\circ} \mathrm{C}$. The sample was scanned and observed under minimal dose condition at $-172^{\circ} \mathrm{C}$. The micrographs were recorded by a Gatan 832 CCD camera at a nominal magnification of 10,000 50,000× and at the defocus of 3-5.46 $\mu \mathrm{m}$.

\section{Amoebae plate test (APT)}

APT was performed as previously described [45]. Briefly, A. castellanii cells were cultured in PYG medium for 3 days prior to the test. A medium change was carried out one day before the test. The amoebae cells were washed off from the tissue culture flask, collected by centrifugation at 2,000 rpm for $5 \mathrm{~min}$ and resuspended in PYG to a density of $2 \times 10^{6} \mathrm{ml}^{-1} .2 \times 10^{6} \mathrm{~A}$. castellanii cells were spread on BCYE agar plates, and incubated at room temperature overnight. Series of tenfold dilutions of stationary-phase bacterial cultures at a starting 
density of $1 \mathrm{OD}_{600}\left(=10^{9}\right.$ cells ml $\left.\mathrm{m}^{-1}\right)$ were prepared. 10 $\mu$ of each dilution were spotted onto the amoebaeCYET agar plates, and incubated at $37^{\circ} \mathrm{C}$ for 5 days.

\section{Cytotoxicity assay using $A$. castellanii}

To determine cytotoxicity, $2.5 \times 10^{5}$ amoebae cells were infected by bacteria at a multiplicity of infection (MOI) of 100. $24 \mathrm{~h}$ post infection, propidium iodide (PI) was added to $3 \mathrm{mg} \mathrm{ml}^{-1}$. A. castellanii cells were detached from the wells and $2.5 \times 10^{4}$ infected amoebae per sample were analyzed using a FACSCalibur flow cytometer (Becton Dickinson) with a scatter gate adjusted for $A$. castellanii [13]. Excitation was at $458 \mathrm{~nm}$ and fluorescence was measured at $495 \mathrm{~nm}$. The data were collected and analyzed using the CELLQUEST software (Becton Dickinson). For fluorescence microscopy, the infected amoebae cells in each well of 24-well plates were stained with PI, then observed in bright field or by epifluorescence with an inverse microscope (Zeiss Axiovert 200 M, $20 \times$ objective).

\section{Intracellular growth in $A$. castellanii}

For intracellular growth assays, exponentially growing $A$. castellanii were washed with Ac (A. castellanii) buffer, resuspended in HL5 medium, seeded onto a 24-well plate $\left(2.5 \times 10^{5}\right.$ per well $)$ and were allowed to adhere for 1-2 h. L. pneumophila was grown for $21 \mathrm{~h}$ in AYE broth, diluted in HL5 and used to infect amoebae at an MOI of 10 . The infection was synchronized by centrifugation at $440 \mathrm{~g}$ for $10 \mathrm{~min}$, and the infected amoebae were incubated at $30^{\circ} \mathrm{C}$. Thirty minutes post infection, extracellular bacteria were removed by washing 3 times with warm HL5 medium [13]. At the time points indicated, culture supernatant was removed and the amoebae cells were lysed with $0.04 \%$ Triton. The supernatant and the lysates were combined, and serial dilutions were prepared and aliquots were plated on CYE plates for CFU counting [72].

\section{Statistical analysis}

Basic statistical analyses were performed using Excel, and one-way ANOVA was performed using SPSS followed by a post hoc Student-Newman-Keul's test. The alignment of amino acid sequences was performed using the online ClustalW2 http://www.ebi.ac.uk/Tools/clustalw2.

\section{Abbreviations}

dot: defect in organelle trafficking; icm: intracellular multiplication; mip: macrophage infectivity potentiator; MIF: mature intracellular form; MOI: multiplicity of infection; APT: amoebae plate test

\section{Acknowledgements}

We thank Miss Ling-yan Zhu for kindly helping perform the flow cytometry analysis. This work was supported by the National Natural Science
Foundation of China (No. 30670106, No. 30970123) and the Guangdong Provincial Natural Science Foundation of China (No.06201654) to YJL.

\section{Authors' contributions}

$\mathrm{XHL}$ and $\mathrm{YJL}$ designed the experiments and drafted the manuscript. $\mathrm{XHL}$ performed the experiments. YLZ and YG participated in the design of the study and performed the amoebae infection analysis. XCZ carried out part of molecular cloning work. QFZ carried out the cyro- electron microscope observation. SNZ participated in designing the study and helped to draft the manuscript. All authors read and approved the final manuscript.

Received: 29 September 2009

Accepted: 19 February 2010 Published: 19 February 2010

\section{References}

1. Fraser DW, Tsai TR, Orenstein W, Parkin WE, Beecham HJ, Sharrar RG, Harris J, Mallison GF, Martin SM, McDade JE, Shepard CC, Brachman PS: Legionnaires' disease: description of an epidemic of pneumonia. $N$ Engl J Med 1977, 297:1189-1197.

2. Kaufmann AF, McDade JE, Patton CM, Bennett JV, Skaliy P, Feeley JC, Anderson DC, Potter ME, Newhouse VF, Gregg MB, Brachman PS: Pontiac fever: isolation of the etiologic agent (Legionella pneumophilia) and demonstration of its mode of transmission. Am J Epidemiol 1981, 114:337-347.

3. Andrews HL, Vogel JP, Isberg RR: Identification of linked Legionella pneumophila genes essential for intracellular growth and evasion of the endocytic pathway. Infect Immun 1998, 66:950-958.

4. Brand BC, Sadosky AB, Shuman HA: The Legionella pneumophila icm locus: a set of genes required for intracellular multiplication in human macrophages. Mol Microbiol 1994, 14:797-808.

5. Ninio S, Zuckman-Cholon DM, Cambronne ED, Roy CR: The Legionella $\mathrm{IcmS}-\mathrm{IcmW}$ protein complex is important for Dot/lcm-mediated protein translocation. Mol Microbiol 2005, 55:912-926.

6. Segal G, Feldman M, Zusman T: The Icm/Dot type-IV secretion systems of Legionella pneumophila and Coxiella burnetii. FEMS Microbiol Rev 2005, 29:65-81.

7. Chen J, de-Felipe KS, Clarke M, Lu H, Anderson OR, Segal G, Shuman HA: Legionella effectors that promote nonlytic release from protozoa. Science 2004, 303:1358-1361.

8. Luo ZQ, Isberg RR: Multiple substrates of the Legionella pneumophila Dot/ $\mathrm{Icm}$ system identified by interbacterial protein transfer. Proc Natl Acad Sci USA 2004, 101:841-846.

9. Ninio S, Roy CR: Effector proteins translocated by Legionella pneumophila : strength in numbers. Trends Microbiol 2007, 15:372-380.

10. Hammer BK, Tateda ES, Swanson MS: A two-component regulator induces the transmission phenotype of stationary-phase Legionella pneumophila . Mol Microbiol 2002, 44:107-118.

11. Molofsky AB, Swanson MS: Differentiate to thrive: lessons from the Legionella pneumophila life cycle. Mol Microbiol 2004, 53:29-40.

12. Hales LM, Shuman HA: The Legionella pneumophila rpoS gene is required for growth within Acanthamoeba castellanii. J Bacteriol 1999, 181:4879-89.

13. Tiaden A, Spirig T, Weber SS, Brüggemann H, Bosshard R, Buchrieser C, Hilbi H: The Legionella pneumophila response regulator LqsR promotes host cell interactions as an element of the virulence regulatory network controlled by RpoS and LetA. Cell Microbiol 2007, 9:2903-2920.

14. Garduño RA, Quinn FD, Hoffman PS: HeLa cells as a model to study the invasiveness and biology of Legionella pneumophila. Can J Microbiol 1998, 44:430-440.

15. Garduño RA, Garduño E, Hiltz M, Hoffman PS: Intracellular growth of Legionella pneumophila gives rise to a differentiated form dissimilar to stationary-phase forms. Infect Immun 2002, 70:6273-6283.

16. Brüggemann $H$, Hagman A, Jules $M$, Sismeiro O, Dillies MA, Gouyette C, Kunst F, Steinert M, Heuner K, Coppée JY, Buchrieser C: Virulence strategies for infecting phagocytes deduced from the in vivo transcriptional program of Legionella pneumophila. Cell Microbiol 2006, 8:1228-1240.

17. Bachman MA, Swanson MS: RpoS co-operates with other factors to induce Legionella pneumophila virulence in the stationary phase. $\mathrm{Mol}$ Microbiol 2001, 40:1201-1214.

18. Bachman MA, Swanson MS: The LetE protein enhances expression of multiple LetA/LetS-dependent transmission traits by Legionella 
pneumophila. Infect Immun 2004, 72:3284-3293.

19. Molofsky AB, Swanson MS: Legionella pneumophila CsrA is a pivotal repressor of transmission traits and activator of replication. Mol Microbiol 2003, 50:445-461.

20. Rasis M, Segal G: The LetA-RsmYZ-CsrA regulatory cascade, together with RpoS and PmrA, post-transcriptionally regulates stationary phase activation of Legionella pneumophila Icm/Dot effectors. Mol Microbiol 2009, 72:995-1010.

21. Sahr T, Brüggemann $H$, Jules $M$, Lomma M, Albert-Weissenberger $C$, Cazalet C, Buchrieser C: Two small ncRNAs jointly govern virulence and transmission in Legionella pneumophila . Mol Microbiol 2009, 72:741-762.

22. Gal-Mor O, Segal G: Identification of CpxR as a positive regulator of $\mathrm{icm}$ and dot virulence genes of Legionella pneumophila. J Bacteriol 2003, 185:4908-4919.

23. Altman $\mathrm{E}$, Segal $\mathrm{G}$ : The response regulator CpxR directly regulates expression of several Legionella pneumophila icm/dot components as well as new translocated substrates. J Bacteriol 2008, 190:1985-1996.

24. Bachman MA, Swanson MS: Genetic evidence that Legionella pneumophila RpoS modulates expression of the transmission phenotype in both the exponential phase and the stationary phase. Infect Immun 2004, 72:2468-2476.

25. Hengge R, Bukau B: Proteolysis in prokaryotes: protein quality control and regulatory principles. Mol Microbiol 2003, 49:1451-1462.

26. Jenal U, Hengge-Aronis R: Regulation by proteolysis in bacterial cells. Curr Opin Microbiol 2003, 6:163-172.

27. Yu AY, Houry WA: ClpP: a distinctive family of cylindrical energydependent serine proteases. FEBS Lett 2007, 581:3749-3757.

28. Gottesman S: Proteolysis in bacterial regulatory circuits. Annu Rev Cell Dev Biol 2003, 19:565-587.

29. Gerth U, Krüger E, Derré I, Msadek T, Hecker M: Stress induction of the Bacillus subtilis clpP gene encoding a homologue of the proteolytic component of the Clp protease and the involvement of ClpP and ClpX in stress tolerance. Mol Microbiol 1998, 28:787-802.

30. Porankiewicz J, Wang J, Clarke AK: New insights into the ATP-dependent Clp protease: Escherichia coli and beyond. Mol Microbiol 1999, 32:449-458.

31. Butler SM, Festa RA, Pearce MJ, Darwin KH: Self-compartmentalized bacterial proteases and pathogenesis. Mol Microbiol 2006, 60:553-562.

32. Frees D, Savijoki K, Varmanen P, Ingmer H: Clp ATPases and ClpP proteolytic complexes regulate vital biological processes in low GC, Gram-positive bacteria. Mol Microbiol 2007, 63:1285-1295.

33. Tomoyasu T, Ohkishi T, Ukyo Y, Tokumitsu A, Takaya A, Suzuki M, Sekiya K, Matsui H, Kutsukake K, Yamamoto T: The ClpXP ATP-dependent protease regulates flagellum synthesis in Salmonella enterica serovar typhimurium. J Bacteriol 2002, 184:645-653.

34. Gaillot O, Pellegrini E, Bregenholt S, Nair S, Berche P: The ClpP serine protease is essential for the intracellular parasitism and virulence of Listeria monocytogenes. Mol Microbiol 2000, 35:1286-1294.

35. Frees D, Qazi SN, Hill PJ, Ingmer H: Alternative roles of ClpX and ClpP in Staphylococcus aureus stress tolerance and virulence. Mol Microbiol 2003, 48:1565-1578

36. Frees D, Chastanet A, Qazi S, Sorensen K, Hill P, Msadek T, Ingmer H: Clp ATPases are required for stress tolerance, intracellular replication and biofilm formation in Staphylococcus aureus. Mol Microbiol 2004, 54:1445-1462.

37. Lemos JA, Burne RA: Regulation and physiological significance of ClpC and ClpP in Streptococcus mutans. J Bacteriol 2002, 184:6357-6366.

38. Wang C, Li M, Dong D, Wang J, Ren J, Otto M, Gao Q: Role of ClpP in biofilm formation and virulence of Staphylococcus epidermidis. Microbes Infect 2007, 9:1376-1383.

39. Maurizi MR, Clark WP, Katayama Y, Rudikoff S, Pumphrey J, Bowers B, Gottesman S: Sequence and structure of ClpP, the proteolytic component of the ATP-dependent Clp protease of Escherichia coli . J Biol Chem 1990, 265:12536-12545.

40. Wang J, Hartling JA, Flanagan JM: The structure of ClpP at $2.3 \mathrm{~A}$ resolution suggests a model for ATP-dependent proteolysis. Cell 1997 91:447-456.

41. LeBlanc JJ, Davidson RJ, Hoffman PS: Compensatory functions of two alkyl hydroperoxide reductases in the oxidative defense system of Legionella pneumophila . J Bacteriol 2006, 188:6235-6244
42. Catrenich $C E$, Johnson W: Characterization of the selective inhibition of growth of virulent Legionella pneumophila by supplemented MuellerHinton medium. Infect Immun 1989, 57:1862-1864.

43. Sadosky AB, Wiater LA, Shuman HA: Identification of Legionella pneumophila genes required for growth within and killing of human macrophages. Infect Immun 1993, 61:5361-5373.

44. Byrne B, Swanson MS: Expression of Legionella pneumophila virulence traits in response to growth conditions. Infect Immun 1998, 66:3029-3034.

45. Albers $U$, Reus $K$, Shuman $H A$, Hilbi $H$ : The amoebae plate test implicates a paralogue of IpxB in the interaction of Legionella pneumophila with Acanthamoeba castellanii. Microbiology 2005, 151:167-182.

46. Berger $\mathrm{KH}$, Isberg RR: Two distinct defects in intracellular growth complemented by a single genetic locus in Legionella pneumophila. Mol Microbiol 1993, 7:7-19.

47. Vogel JP, Isberg RR: Cell biology of Legionella pneumophila . Curr Opin Microbiol 1999, 2:30-34

48. Cooke MS, Evans MD, Dizdaroglu M, Lunec J: Oxidative DNA damage: mechanisms, mutation, and disease. FASEB J 2003, 17:1195-1214.

49. Xiao H, Li TK, Yang JM, Liu LF: Acidic pH induces topoisomerase IImediated DNA damage. Natl Acad Sci USA 2003, 100:5205-5210.

50. Gonzalez M, Rasulova F, Maurizi MR, Woodgate R: Subunit-specific degradation of the UmuD/D' heterodimer by the ClpXP protease: the role of trans recognition in UmuD' stability. EMBO J 2000, 19:5251-5258.

51. Michel A, Agerer F, Hauck CR, Herrmann M, Ullrich J, Hacker J, Ohlsen K: Global regulatory impact of ClpP protease of Staphylococcus aureus on regulons involved in virulence, oxidative stress response, autolysis, and DNA repair. J Bacteriol 2006, 188:5783-5796.

52. Savijoki K, Ingmer $H$, Frees D, Vogensen FK, Palva A, Varmanen P: Heat and DNA damage induction of the LexA-like regulator HdiR from Lactococcus lactis is mediated by RecA and ClpP. Mol Microbiol 2003, 50:609-621.

53. Msadek T, Dartois V, Kunst F, Herbaud ML, Denizot F, Rapoport G: ClpP of Bacillus subtilis is required for competence development, motility, degradative enzyme synthesis, growth at high temperature and sporulation. Mol Microbiol 1998, 27:899-914.

54. Jenal U, Fuchs T: An essential protease involved in bacterial cell-cycle control. EMBO J 1998, 17:5658-5669.

55. Nair S, Poyart C, Beretti JL, Veiga-Fernandes H, Berche P, Trieu-Cuot P: Role of the Streptococcus agalactiae ClpP serine protease in heat-induced stress defence and growth arrest. Microbiology 2003, 149:407-417.

56. Kock H, Gerth $U$, Hecker M: MurAA, catalysing the first committed step in peptidoglycan biosynthesis, is a target of Clp-dependent proteolysis in Bacillus subtilis. Mol Microbiol 2004, 51:1087-1102.

57. Weart RB, Nakano S, Lane BE, Zuber P, Levin PA: The ClpX chaperone modulates assembly of the tubulin-like protein FtsZ. Mol Microbiol 2005, 57:238-249.

58. Margolin W: FtsZ and the division of prokaryotic cells and organelles. Nat Rev Mol Cell Biol 2005, 6:862-871.

59. Hovel-Miner G, Pampou S, Faucher SP, Clarke M, Morozova I, Morozov P, Russo JJ, Shuman HA, Kalachikov S: SigmaS controls multiple pathways associated with intracellular multiplication of Legionella pneumophila . J Bacteriol 2009, 191:2461-2473.

60. Ibrahim YM, Kerr AR, Silva NA, Mitchell TJ: Contribution of the ATPdependent protease ClpCP to the autolysis and virulence of Streptococcus pneumoniae. Infect Immun 2005, 73:730-740.

61. Hengge-Aronis R: Signal transduction and regulatory mechanisms involved in control of the sigma(S) (RpoS) subunit of RNA polymerase. Microbiol Mol Biol Rev 2002, 66:373-395.

62. Jackson MW, Silva-Herzog E, Plano GV: The ATP-dependent ClpXP and Lon proteases regulate expression of the Yersinia pestis type III secretion system via regulated proteolysis of $\mathrm{YmoA}$, a small histone-like protein. Mol Microbiol 2004, 54:1364-1378.

63. Loughlin MF, Arandhara V, Okolie C, Aldsworth TG, Jenks PJ: Helicobacter pylori mutants defective in the ClpP ATP-dependant protease and the chaperone CIpA display reduced macrophage and murine survival. Microb Pathog 2009, 46:53-57.

64. Sexton JA, Miller JL, Yoneda A, Kehl-Fie TE, Vogel JP: Legionella pneumophila DotU and $\mathrm{IcmF}$ are required for stability of the Dot/lcm complex. Infect Immun 2004, 72:5983-5992. 
65. Hammer BK, Swanson MS: Co-ordination of Legionella pneumophila virulence with entry into stationary phase by ppGpp. Mol Microbiol 1999, 33:721-731.

66. Segal G, Shuman HA: Legionella pneumophila utilizes the same genes to multiply within Acanthamoeba castellanii and human macrophages. Infect Immun 1999, 67:2117-2124.

67. Sambrook J, Russell DW: Molecular Cloning: a Laboratory Manual Cold Spring Harbor, Cold Spring Harbor Press 2001.

68. Ho SN, Hunt HD, Horton RM, Pullen JK, Pease LR: Site-directed mutagenesis by overlap extension using the polymerase chain reaction. Gene 1989, 77:51-59.

69. Yu HB, Zhang YL, Lau YL, Yao F, Vilches S, Merino S, Tomas JM, Howard SP, Leung KY: Identification and characterization of putative virulence genes and gene clusters in Aeromonas hydrophila PPD134/91. Appl Environ Microbiol 2005, 71:4469-4477.

70. Köhler R, Bubert A, Goebel W, Steinert M, Hacker J, Bubert B: Expression and use of the green fluorescent protein as a reporter system in Legionella pneumophila. Mol Gen Genet 2000, 262:1060-1069.

71. Chen DQ, Zheng XC, Lu YJ: Identification and characterization of novel ColE1-type, high-copy number plasmid mutants in Legionella pneumophila . Plasmid 2006, 56:167-178.

72. Al-Khodor S, Price CT, Habyarimana F, Kalia A, Abu Kwaik Y: A Dot/lcmtranslocated ankyrin protein of Legionella pneumophila is required fo intracellular proliferation within human macrophages and protozoa. Mol Microbiol 2008, 70:908-923.

doi:10.1186/1471-2180-10-54

Cite this article as: Li et al:: The ClpP protease homologue is required for the transmission traits and cell division of the pathogen Legionella pneumophila. BMC Microbiology 2010 10:54.

\section{Submit your next manuscript to BioMed Central and take full advantage of:}

- Convenient online submission

- Thorough peer review

- No space constraints or color figure charges

- Immediate publication on acceptance

- Inclusion in PubMed, CAS, Scopus and Google Scholar

- Research which is freely available for redistribution

Submit your manuscript at www.biomedcentral.com/submit 